\title{
Maps for electron cloud density in Large Hadron Collider dipoles
}

\author{
T. Demma \\ Istituto Nazionale di Fisica Nucleare (INFN), Laboratori Nazionali di Frascati, Via E. Fermi 40, I-00044 Frascati (Rome), Italy \\ S. Petracca \\ Department of Engineering, University of Sannio at Benevento, C.so Garibaldi 107, I-80125 Benevento, Italy
}

F. Ruggiero, ${ }^{*}$ G. Rumolo, and F. Zimmermann

European Organization for Nuclear Research (CERN), CH-1211 Genève 23, Switzerland

(Received 10 March 2007; published 26 November 2007)

\begin{abstract}
The generation of a quasistationary electron cloud inside the beam pipe through beam-induced multipacting processes has become an area of intensive study. The analyses performed so far have been based on heavy computer simulations taking into account photoelectron production, secondary emission, electron dynamics, and space charge effects, providing a detailed description of the electroncloud evolution. Iriso and Peggs [U. Iriso and S. Peggs, Phys. Rev. ST Accel. Beams 8, 024403 (2005)] have shown that, for the typical parameters of RHIC, the bunch-to-bunch evolution of the average electron-cloud density at a point can be represented by a cubic map. Simulations based on this map formalism are orders of magnitude faster compared to those based on standard particle tracking codes. In this communication we show that the map formalism is also applicable to the case of the Large Hadron Collider (LHC), and that, in particular, it reproduces the average electron-cloud densities computed using a reference code to within $\sim 15 \%$ for general LHC bunch filling patterns. We also illustrate the dependence of the polynomial map coefficients on the physical parameters affecting the electron cloud (secondary emission yield, bunch charge, bunch spacing, etc.).
\end{abstract}

DOI: $10.1103 /$ PhysRevSTAB.10.114401

PACS numbers: 29.20.Dh, 29.27.-a

\section{INTRODUCTION}

Photoemission and/or ionization of the residual gas in the beam pipe produces electrons, which move under the action of the beam field and their own space charge. These primary electrons may initiate the buildup of a quasistationary electron cloud, which can severely affect the machine operation.

A number of sophisticated computer simulation codes, e.g., PEI [1], POSINST [2], and ECLOUD [3], have been developed to study the e-cloud effect, and their predictions have been compared with experimental observations.

Depending on the problem's (beam and pipe) parameters, these codes simulate some $10^{10}$ electrons per meter. Typically, they track macroparticles comprising up to a maximum of $\sim 10^{5}$ electrons each, taking into account all forces acting upon them. As macroparticles produce more electrons due to secondary emission, their total charge is increased, and/or additional macroparticles are included. A large amount of CPU time is accordingly needed: a complete e-cloud simulation can last from a few hours to several days.

In the cases studied here (for the parameter values summarized in Table I), a single simulation lasts about 12 hours on a Pentium-IV class workstation.

A new perspective was suggested in [4], where it was noted that the evolution of the electron density at a point,

*Deceased. averaged over the time interval between successive bunch passages, could be accurately described by a simple (cubic) map.

Such an approach is ultimately useful because the map is easily computable, and allows a quick scan of some key design parameters, such as the bunch filling pattern, while effectively boiling down the detailed description of the underlying physics, including the secondary emission yield (henceforth SEY), the quantum efficiency, etc., into a few effective parameters. On the other hand, the map gives information about the behavior of the e-cloud evolution

TABLE I. Input parameters for ECLOUD simulations.

\begin{tabular}{lcc}
\hline \multicolumn{1}{c}{ Parameter } & Units & Value \\
\hline Beam particle energy & $\mathrm{GeV}$ & 7000 \\
Bunch spacing & $\mathrm{m}$ & 7.48 \\
Bunch length & $\mathrm{m}$ & 0.075 \\
Number of bunches $N_{b}$ & & 72 \\
Number of particles per bunch $N$ & & $8 \times 10^{10}$ to $1.4 \times 10^{11}$ \\
Bending field $B$ & $\mathrm{~T}$ & 8.4 \\
Length of bending magnet & $\mathrm{m}$ & 1 \\
Vacuum screen half height & $\mathrm{m}$ & 0.018 \\
Vacuum screen half width & $\mathrm{m}$ & 0.022 \\
Circumference & $\mathrm{m}$ & 27000 \\
Primary photoemission yield & & $8 \times 10^{-4}$ \\
Maximum SEY, $\delta_{\text {max }}$ & & 1.3 to 1.7 \\
Energy for maximum SEY, $E_{\max }$ & $\mathrm{eV}$ & 237 \\
Energy width of secondary $e^{-}$ & $\mathrm{eV}$ & 1.8 \\
\hline \hline
\end{tabular}


at a single specific point along the ring, other locations being characterized by different map parameters.

The validity of the map description was demonstrated in [4] for the specific case of RHIC [5]. It is obviously interesting to investigate the applicability of the map formalism to different machines, like the Large Hadron Collider (LHC), for which the e-cloud problem is also particularly important.

In this paper we show that a simple cubic map can indeed be effective in modeling the bunch-to-bunch evolution of the electron-cloud density in the LHC arc dipoles, and for identifying the bunch filling patterns for which the e-cloud saturation density does not exceed some critical threshold.

The paper is accordingly laid out as follows. In Sec. II a map model for the average e-cloud density evolution in the LHC is introduced and discussed. In Sec. III it is shown that the map whose parameters have been fitted to the numerical simulations run on a particular filling pattern, describes equally well the e-cloud density evolution corresponding to general bunch filling patterns. The map is accordingly used to analyze the e-cloud evolution for several different bunch filling patterns.

Hints for future work are listed in the Conclusions.

\section{THE CUBIC MAPS}

The typical time evolution of the electron-cloud line density at some point of an LHC arc dipole, computed by ECLOUD, is shown in Fig. 1, for a filling pattern consisting of a train of 72 bunches followed by gaps of 28 empty (zero charge) bunches, and the beam/pipe parameters collected in Table I [6].

Except for superimposed oscillations, the density grows exponentially in time as more and more bunches pass by, until saturation occurs. The subsequent decay corresponds to the successive passage of the empty bunch train.

By averaging the density in the intervals between successive bunch passages, we obtain the markers in Fig. 1.

From Fig. 1, one can see that the evolution of the e-cloud density averaged in the intervals from a bunch passage to the next can be used to track the buildup and decay regimes, although the details of the oscillations of the ecloud density between successive bunches are lost.

Figure 2 shows the behavior of the averaged electron density (computed as explained above) after the passage of bunch $m$, denoted as $\rho_{m+1}$, as a function of $\rho_{m}$, for two different values of the bunch charge, $N$ (green and blue circle markers). The dashed red line in Fig. 2 corresponds to saturation (fixed points of the $\rho_{m} \rightarrow \rho_{m+1}$ map).

As more and more bunches pass by, the initially small electron-cloud density builds up (points above the red line, $\rho_{m+1}>\rho_{m}$ ), eventually approaching saturation. In the saturation regime, the $\rho_{m}$ tend to cluster along the red line. Points below the red line $\left(\rho_{m+1}<\rho_{m}\right)$ describe the

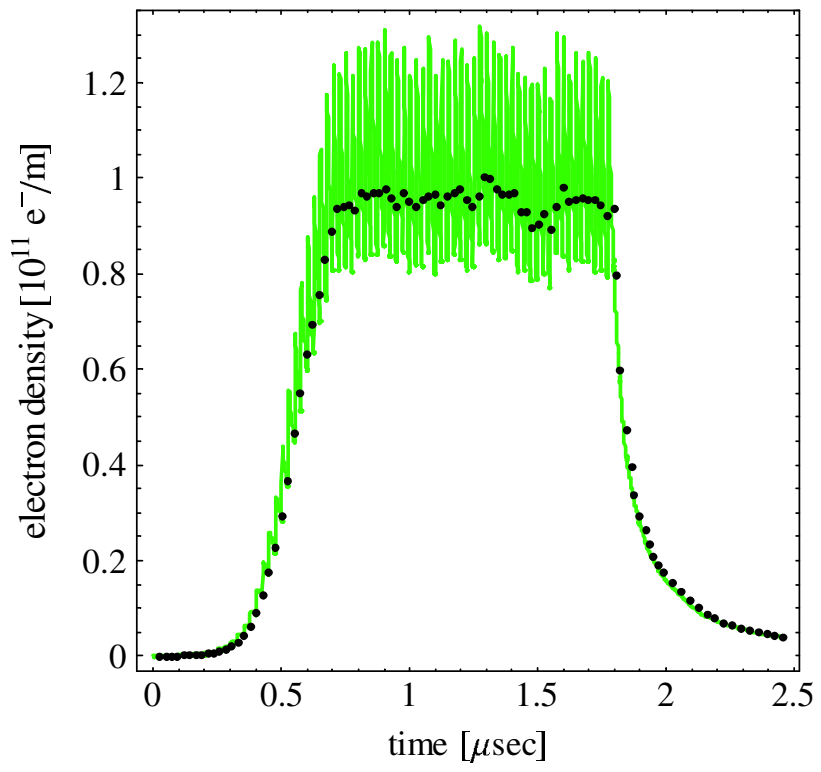

FIG. 1. (Color) Evolution of the electron density (green line) computed with ECLOUD. The case shown corresponds to a filling pattern featuring 72 charged bunches, with bunch charge of $N=$ $1.0 \times 10^{11}$ protons, followed by 28 empty (zero-charge) bunches. The assumed bunch spacing is $7.48 \mathrm{~m}$, and the SEY is $\delta_{\max }=1.5$. The black markers identify the average electron density between two consecutive bunches.

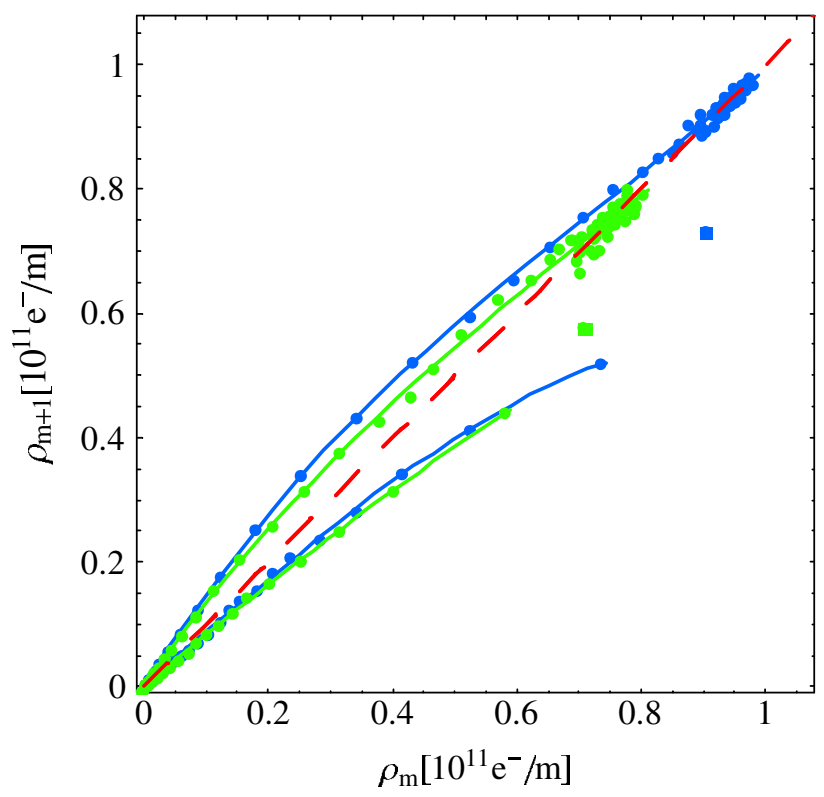

FIG. 2. (Color) Average e-cloud density map $\rho_{m+1}$ vs $\rho_{m}$. Circle markers: ECLOUD simulations $\left(\delta_{\max }=1.7\right.$, all other parameters as in Table I). The dashed red line represents saturation $\left(\rho_{m+1}=\right.$ $\left.\rho_{m}\right)$. Markers above the saturation line describe the e-cloud buildup (cyan: $N=1.6 \times 10^{11}$; green: $N=0.8 \times 10^{11}$ ). Markers below the saturation line describe the e-cloud decay. The cyan and green lines are the corresponding cubic fits. Transitions between filled and empty bunch trains are shown as square markers. 
decay regime. The decay regime, which corresponds to the passing by of the empty bunches is, more or less obviously, almost independent of $N$.

The continuous curves in Fig. 2 correspond to homogeneous cubic fits,

$$
\rho_{m+1}=a \rho_{m}+b \rho_{m}{ }^{2}+c \rho_{m}{ }^{3}
$$

which are seen to reproduce the data quite well. The map idea introduced in [4] with reference to RHIC thus works also for LHC.
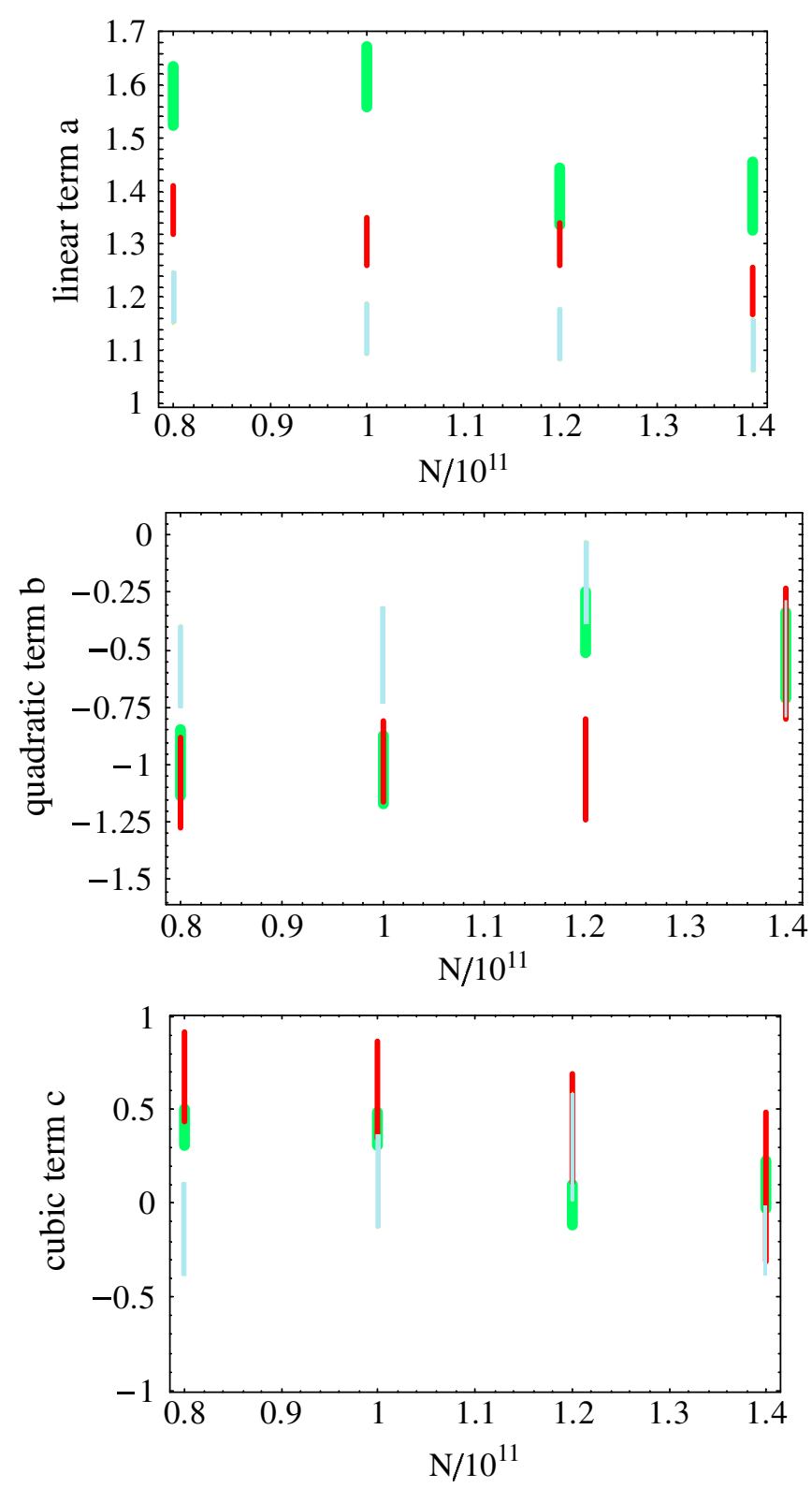

FIG. 3. (Color) The $a, b, c$ coefficients obtained by fitting the map (1) to the density buildup regime, for several $N$. The bars represent the $95 \%$ confidence intervals for $\delta_{\max }=1.3$ (cyan), $\delta_{\max }=1.5$ (red), and $\delta_{\max }=1.7$ (green). All other parameters as in Table I.
The only exceptions are represented by the transitions between filled and empty bunch subtrains, represented by the square markers in Fig. 2. This is not unexpected, and was already noted in [4].

The three terms in the map (1) describe, respectively, the exponential growth/decay mechanism (linear term, larger/ smaller than 1, respectively), the space charge effects leading to saturation (quadratic term, whose sign reflects the concavity of the curves), and an additional correction (cubic term) embodying small corrections.

At present, there is only partial clear physical insight into the dependence of the above map parameters on the problem's (beam and pipe) configuration, and their values must be deduced empirically from numerical simulations.

Once the coefficients have been determined, however, the model is accurate for all filling patterns, as further illustrated in Sec. III.

In Fig. 3 the values of the linear, parabolic, and cubic coefficients in the map (1) which fits the e-cloud density buildup regime are plotted for different SEY values (1.3, 1.5 , and 1.7) and a number of values of $N$. The pertinent 95\% confidence intervals are also shown. The linear coefficient is a monotonic increasing function of the SEY, and its dependence on $N$ is approximately linear, for $\delta_{\max }$ not too large. The parabolic term is always negative. The cubic term can have any sign, and is consistently smaller than the parabolic one.

\section{FILLING PATTERNS}

The buildup of the e-cloud can be controlled by inserting empty bunch subtrains in the charged bunch trains [7]. It is important to have efficient (i.e., fast and accurate) tools to compute the maximum (saturation) density of the e-cloud corresponding to different bunch-train filling patterns, to identify those yielding tolerable electron-cloud densities. This problem is particularly relevant for LHC [8].

The map formalism is quite fast and sufficiently accurate for this purpose [9]. The key property of the map is that, for all practical purposes, its coefficients do not depend on the bunch filling pattern, and thus can be deduced from a single time-consuming simulation.

As an illustration of this important property, we use the map coefficients corresponding to the reference filling pattern of LHC (72 charged bunches) to predict the (averaged) e-cloud density evolution for different filling patterns, and compare the result to those obtained from ECLOUD.

For all cases discussed below, we keep the total length of both the bunch trains and the gaps separating successive bunch trains fixed at the LHC reference values (72 and 28 bunch lengths, respectively), and change only the bunchtrain filling pattern.

The agreement between the ECLOUD and map predictions is quite good, as seen from Fig. 4 (displaying the $\rho_{m+1}$ vs $\rho_{m}$ map) and Fig. 5 (displaying the time evolution, 


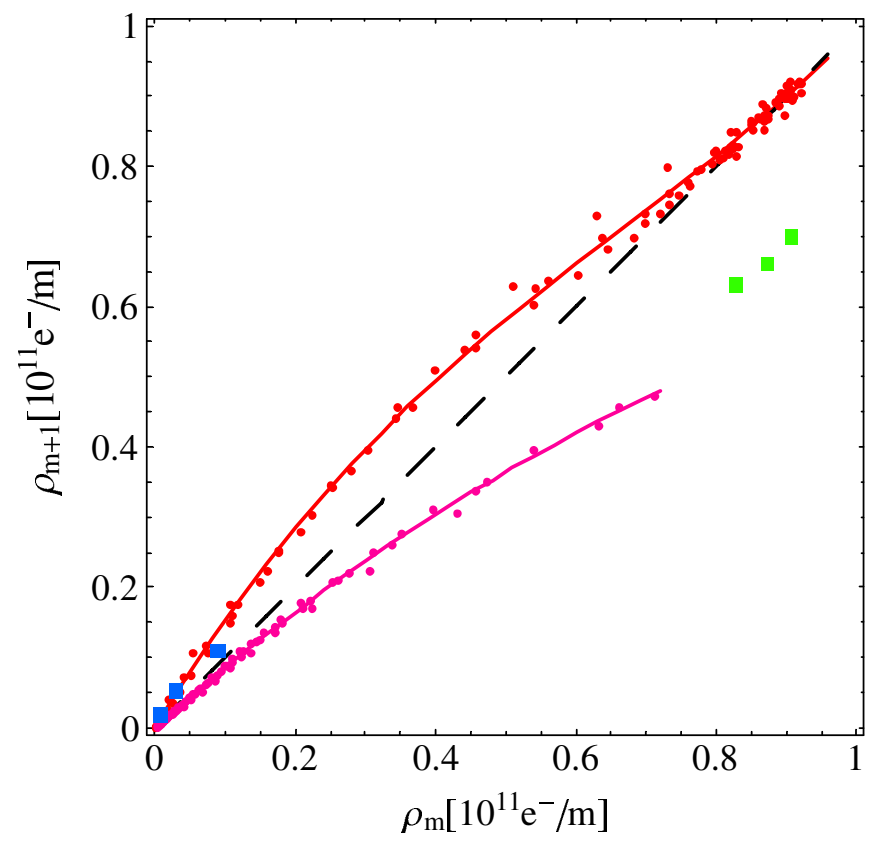

FIG. 4. (Color) Average e-cloud density map $\rho_{m+1}$ vs $\rho_{m}$. The circle markers correspond to the ECLOUD computed values for the $24 f-12 e-36 f$ filling pattern. The lines correspond to the predictions of the map whose parameters have been fitted to the ECLOUD simulation run for the $72 f$ (LHC reference) filling pattern. In both cases, successive bunch trains are separated by gaps whose length corresponds to 28 (empty) bunches (LHC reference). All other parameters have the values in Fig. 1. The square markers correspond to the transitions between charged and empty bunch subtrains. They can also be fitted by cubic maps, characterized by different coefficients.

$\rho_{m}$ vs $m$ ). The relative error does not exceed $15 \%$ in the worst case. On the other hand, the reduction in the computing time is several orders of magnitude: ECLOUD needs about $12 \mathrm{~h}$ for each simulation (for the parameters listed in Table I) on a Pentium IV workstation, while the map takes a fraction of a second.

Thus, the e-cloud peak densities corresponding to different filling patterns can be readily computed.

As an illustration, the peak values of the e-cloud density computed using the cubic map for several filling patterns have been collected in Table II. The corresponding values

TABLE II. Peak (saturation) value of the (linear) electroncloud density (in units of $\left[10^{11} e^{-} / m\right]$ ) in the LHC arc dipoles for different filling patterns. Comparison between ECLOUD and map results.

\begin{tabular}{lccc}
\hline \hline \multicolumn{1}{c}{ Filling } & Map & ECLOUD & Error $(\%)$ \\
\hline Reference $(72 f)$ & 1.01 & 1.03 & 1.9 \\
$(24 f, 12 e, 36 f)$ & 0.95 & 1.01 & 5.9 \\
$(24 f, 24 e, 24 f)$ & 0.95 & 0.98 & 3.1 \\
$3 \times(12 f, 12 e)$ & 0.81 & 0.80 & 1.2 \\
$6 \times(6 f, 6 e)$ & 0.63 & 0.62 & 1.6 \\
\hline \hline
\end{tabular}

obtained from ECLOUD are also shown for comparison, together with the relative percentage error.

The results in Table II show that for a total number of 36 filled bunches the peak e-cloud density can be reduced by $\sim 40 \%$ going from $(36 f, 36 e)$ to $6 \times(6 f, 6 e)$ filling pattern. The following intuitive scenario is confirmed: the total number of bunches in each filled subtrain should be
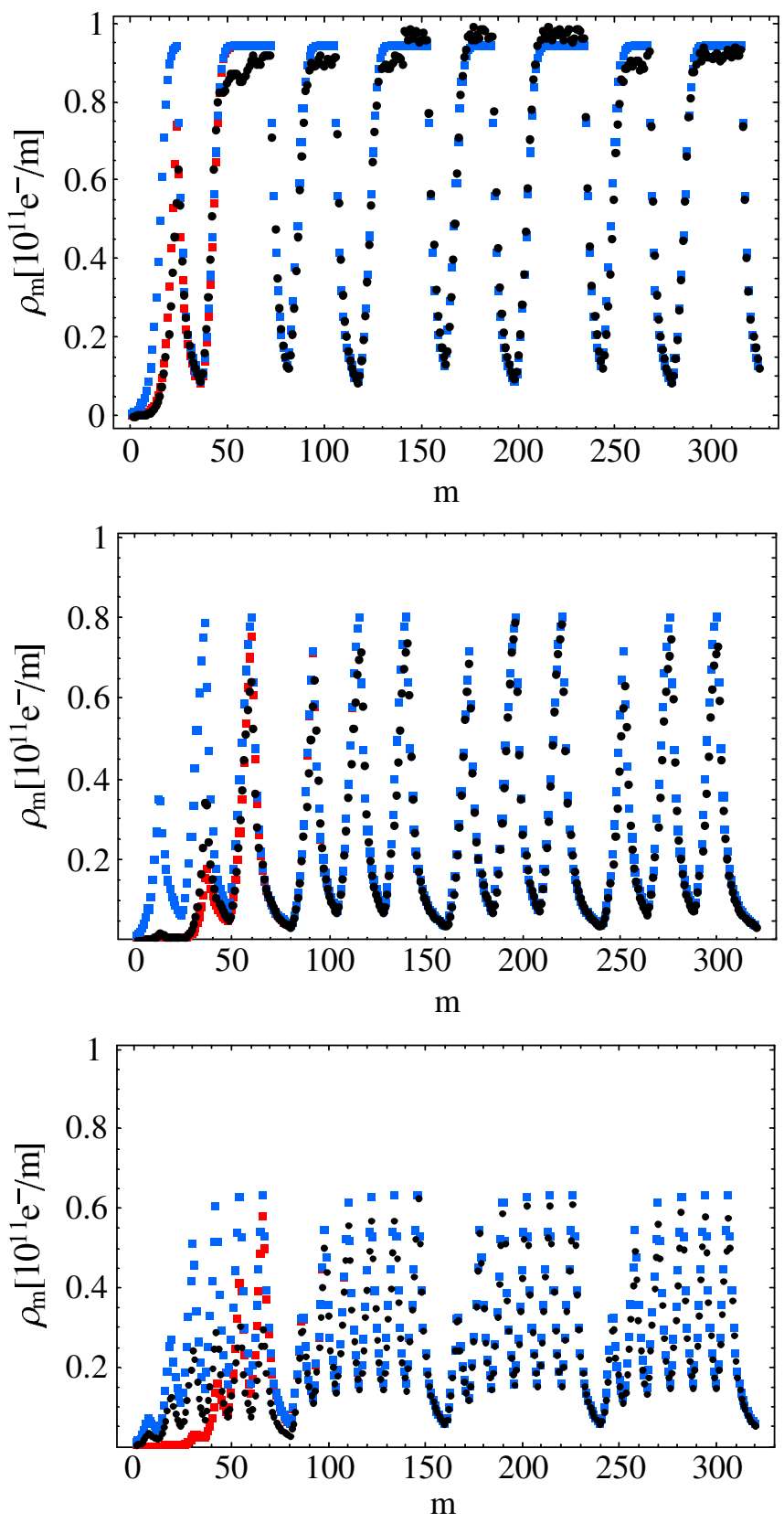

FIG. 5. (Color) Average e-cloud density evolution for three different bunch-train filling patterns, for $N=1.2 \times 10^{11}$ and $\delta_{\max }=1.7$. Top: $24 f-12 e-36 f$; mid: $3 \times(12 f-12 e)$; bottom: $6 \times(6 f-6 e)$. In both cases, successive bunch trains are separated by gaps whose length corresponds to 28 (empty) bunches. Black markers: ECLOUD results. Blue and red markers: map results corresponding to initial electron densities $\rho_{0}=10^{9}$ and $\rho_{0}=10^{7}\left[e^{-} / \mathrm{m}\right]$, respectively. 
small enough to prevent saturation, while the total number of empty bunches in each empty subtrain should be large enough to allow for almost complete charge decay, to prevent buildup. Neither condition alone is sufficient. Both conditions can be fulfilled at the expense of some reduction in the luminosity.

\section{CONCLUSIONS AND OUTLOOK}

The electron-cloud buildup in the LHC dipoles can be described by a cubic map. The coefficients of this map depend on the pipe and beam parameters, and can be simply deduced from e-cloud simulation codes modeling the involved physics in full detail. Remarkably, if all other parameters (namely, the bunch charge $N$, the SEY, and the pipe parameters) are held fixed, the map coefficients basically do not depend on the filling pattern.

The map can be thus used as a quick and (not so) dirty tool for finding filling patterns yielding electron-cloud densities compatible with safe operation.

Unfortunately, at present, no physical model for relating (even in an approximate and/or phenomenological way) all map coefficients to the problem's parameter is available. In this connection, any progress toward deeper insight would be significant. In his thesis ([10], chapter 10), Iriso developed a simple though detailed (only electron rediffusion is neglected) analytic model for the linear coefficient, exploiting its dependency on chamber radius, bunch spacing, maximum SEY, zero-energy reflection probability, bunch intensity, and length. Iriso's model agrees well with simulations for the specific case of RHIC. We are working toward adapting his approach to the LHC case, and comparing results to numerical simulations.

Possible generalizations of the map formalism could be the inclusion of heat load and electron flux at the chamber wall, e.g., for the exploration of LHC conditioning scenarios [11]. Including the scrubbing history, whose importance for e-clouds has been recently emphasized [11], in the big simulation codes would most likely result in improved accuracy of the derived maps. Work in all these directions is planned or in progress.

\section{ACKNOWLEDGMENTS}

Sadly Francesco Ruggiero passed away in January 2007. This work was completed under his continued inspiration and guidance.
[1] K. Ohmi, Phys. Rev. Lett. 75, 1526 (1995).

[2] M. A. Furman and G. R. Lambertson, in Proceedings of the International Workshop on Multibunch Instabilities in Future Electron and Positron Accelerators: MBI-97, Tsukuba, Japan, 1997, edited by Y.H. Chin, KEK Proceedings No. 97-17 (High Energy Accelerator Research Organization, Tsukuba, Japan, 1997), p. 170; M. A. Furman and M.T.F. Pivi, Phys. Rev. ST Accel. Beams 5, 124404 (2002).

[3] F. Zimmermann, CERN, LHC-Project-Report-95, 1997; G. Rumolo and F. Zimmermann, CERN, SL-Note-2002016, 2002; see also code web site http://wwwslap.cern.ch/ electron-cloud/Programs/Ecloud/ecloud.html.

[4] U. Iriso and S. Peggs, in Proceedings of the 9th European Particle Accelerator Conference, edited by L. Rivkin et al. (European Physical Society, Accelerator Group and CERN, 2004), p. 2870.

[5] The map technique also allows one to build a simple coupled model including ions and electrons [12], and to explore qualitatively the various phases of the coupled electron-ion system.

[6] The parameters in Table I are those typical of an LHC dipole. We use the SEY model described in [13], and an elastic reflection probability at the zero electron energy scaling factor of 0.5 [14].

[7] R. Cappi et al., Phys. Rev. ST Accel. Beams 5, 094401 (2002).

[8] G. Rumolo, F. Ruggiero, and F. Zimmermann, Phys. Rev. ST Accel. Beams 4, 012801 (2001); 4, 029901 (2001).

[9] Th. Demma, S. Petracca, F. Ruggiero, G. Rumolo, and F. Zimmermann, in Proceedings of the 10th European Particle Accelerator Conference, edited by C. Biscari et al. (European Physical Society, Accelerator Group and CERN, 2006), p. 2889.

[10] U. Iriso, Ph.D. thesis, University of Barcelona, 2005.

[11] D. Schulte and F. Zimmermann, in Proceedings of the 31st Advanced ICFA Beam Dynamics Workshop on ElectronCloud Effects: ECLOUD04, Napa, California, 2004, edited by M. Furman, S. Henderson, and F. Zimmermann (CERN Yellow Report No. CERN-2005001), 2005, p. 143.

[12] U. Iriso and S. Peggs, Phys. Rev. ST Accel. Beams 9, 071002 (2006).

[13] R. Cimino et al., Phys. Rev. Lett. 93, 014801 (2004).

[14] D. Schulte et al., in Proceedings of the Particle Accelerator Conference (PAC'05), edited by C. Horak (IEEE Press, Piscataway, NJ, 2005), p. 1371. 